\title{
A Continuation Method in the Teaching of Academic English Writing for Chinese College Students
}

\author{
Lei Gao \\ School of Foreign Languages, Guangdong University of Petrochemical Technology, Maoming, Guangdong 525000, \\ China \\ Corresponding author. Email: mrgaolei@163.com
}

\begin{abstract}
The 'continuation method' means that people tend to borrow or imitate their interlocutors' language to express ideas or to continue a discourse construction. There were 20 Chinese second-year college students in the study. They were given two tests on academic English writing. In Test 1, the students were required to write a composition of about 200 words according to the given topic. In Test 2, according to the 'continuation method', they were required to make a sequel writing of about 200 words in line with the meaning and stylistic features of the given language material. The results showed that most students did better in Test 2 . The study probed into the mechanism of 'continuation method' in improving the students' academic English writing.
\end{abstract}

Keywords: continuation method; academic English writing; English language teaching

\section{INTRODUCTION}

Chuming Wang ${ }^{[1]}$ pointed out that language is acquired through "continuation", and through "continuation" people can get a high efficiency of language learning. On the promotion function of "continuation" in language acquisition, he published a serial of research articles, and thus his "continuation theory" about language acquisition came into being, which has already exerted influence upon the academic circle in China. Some other scholars, such as Ming Wang, Li Liu and Lei Zhang, have conducted relevant empirical research, confirming the highly effective promotion function of "continuation". Actually, the 'continuation method' is an interactive way of language learning, which can promote a timely communication between interlocutors and improve the idiomaticity of language production, so it can help improve the efficiency of language learning. The "continuation theory" is innovative, and to a certain extent it challenges the innateness hypothesis of language acquisition and makes up for the deficiency of the existing interaction theory. The "continuation theory" advocates direct and timely communication between interlocutors, and it argues that language input and output are not two separated phases but closely interrelated. The operational mechanism of "continuation" lies in its "structural priming" and "alignment". The "structural priming" means that interlocutors, stimulated by language interaction, tend to use the language expressions already used by their partners or themselves ${ }^{[2]}$; the "alignment" means that the language abilities of interlocutors will level out, and thus the language ability of learners will be improved ${ }^{[3]}$. The article investigated two tests of 20 students in academic English writing, probed into how the "continuation method" influenced upon them, and tried to answer the following questions:

1 In what linguistic levels does the continuation method have better alignment?

2 Is the effect of continuation method influenced by the students' language ability?

3 What should we pay attention to when using the continuation method in teaching?

\section{RATIONALE}

There are many relevant theories and hypotheses dealing with how people acquire language, such as: stimulusresponse theory, cognitive theory, behavior theory, innateness hypothesis, contrastive analysis hypothesis, inter-language hypothesis, input hypothesis, hypothesis of universal grammar, hypothesis of cultural adaptation and so on. From the findings of these theories and hypotheses, we know that language acquisition is almost inseparable from language interaction. On the basis of interaction theory, the "continuation theory" advocates that in the process of language interaction, people tend to imitate or borrow the language of their partners to express their own ideas or to achieve certain communicative purposes. On most occasions, there exists a gap in language proficiency between the interlocutors. It is just in the process of bridging this gap that people master the rules of language and their use contexts. So Chuming Wang ${ }^{[4]}$ remarked, "Language is learned through 'continuation'. The reason for why adults find it difficult to learn a foreign language lies in the lack of interaction with good or native speakers and the lack of opportunities for 'continuation' of language".

In fact, the interaction of human language is very important. Otherwise, it may be impossible for people to 
acquire a language even though they have the so-called "innate language acquisition mechanism". Maybe that is why two children became "wolf children", as they were raised by wolves. B. Ambridge et al. ${ }^{[5]}$ pointed out that the frequency of language input and output profoundly affects children's first language acquisition; K. Bergström et al. ${ }^{[6]}$ pointed out that in the process of second language acquisition, the quantity and quality of second language input play an important role; I. Kaiser and G. Kasberger ${ }^{[7]}$ pointed out that children's acquisition of a language is closely related to their parents' degree of education. They remarked that if the parents have a good education background, they can often communicate with their children with more and better language materials. Therefore, we can say that the acquisition of a language is inseparable from the language interaction, and if in the absence of necessary interaction, it will be difficult for people to properly use the relevant language knowledge. In this light, Qiufang Wen ${ }^{[8]}$ pointed out that language knowledge cannot be automatically converted into one's output ability without language interaction. Therefore, the study of language acquisition and language teaching should not only pay attention to the input or acceptance of language, but also the output or use of language. The "continuation theory" focuses on the integration of language input and output and stresses the "alignment" of language interaction. Chuming Wang [9] pointed out, "Whether in foreign language or second language context, the effect of 'continuation' in language learning is both salient", and he figured out the process of "continuation" in language acquisition as Figure 1:

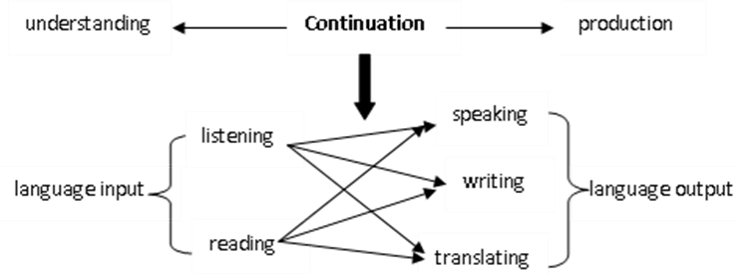

Figure 1: 'continuation' in language acquisition

The figure shows that language learners, driven by the need of "continuation", will make a good understanding of the input language in order to make a good and timely language output, which gets the understanding and production of language closely connected. Language input is realized via listening or reading; language output is realized via speaking, writing or translating. The purpose of listening or reading is for speaking, writing or translating, and all of these language activities are driven by "continuation", which is a mental mechanism of the language learners.

\section{PARTICIPANTS}

Twenty Chinese second-year college students took part in the study. They major in English language and literature, and they have already learned the courses of Advanced English Grammar, Basic English Writing, British and
American Literature, and so on. To verify at what linguistic levels and in what ways the "continuation method" influenced the students' academic English writing, we gave them two tests at the middle of the semester. In Test 1 , they were required to write an English composition of about 200 words according to the given topic, and in Test 2 , according to the "continuation method", they were required to make a sequel writing of about 200 words in line with the meaning and stylistic features of the given English material.

\section{PROCEDURE}

At the middle of the semester, we gave the students two tests to write academic English compositions on Chinese calligraphy. In Test 1, they were asked to write an English composition of about 200 words according to the topic of "Chinese calligraphy". Two days later, they were given Test 2 to make a sequel writing of about 200 words about the same topic in line with the meaning and stylistic features of the given English material. And then, we gave them a questionnaire and investigated how they thought during the two tests. Finally, we made a comparison of the students' two tests, trying to find out at what linguistic levels and in what ways the "continuation method" influenced their academic English writing.

Test 1

Write an English composition of about 200 words on Chinese calligraphy.

Test 2

Make a sequel writing of about 200 words on Chinese calligraphy in line with the meaning and stylistic features of the given English material.

Questionnaire

1 Which writing do you think is more difficult, Test 1 or Test 2?

2 What did you mainly refer to from the given English material in Test 2?

3 Did you think of using the words, expressions or sentence structures written in Test 1 when you did Test 2?

4 What is the main problem about your academic English writing?

5 Do you think the sequel writing is helpful? If so, in what aspects?

\section{RESULTS}

The statistical results showed that the students' writing in Test 1 generally had such problems as follows:

- grammatical mistakes

- too much Chinglish

- inexact words and expressions

- lack of specialized vocabulary.

Let's take one student's writing for example.

There are not only tens of millions of words in the world, but also tens of millions of ways of writing. In fact, there are at most two styles of writing in each country. Chinese 
after 5,000 years of baptism and precipitation, whether it is written on paper or any other carrier can have a different soul. With the development of time, it even formed a relatively complete Chinese calligraphy system. (excerpt) It is easy to find that in this paragraph there are many grammatical mistakes, inexact diction, and Chinglish, so the writing gets nowhere in expressing the student's ideas. Through comparison and statistics, we found that most students made an obvious progress in Test 2, where the main problems that they made in Test 1 were greatly reduced. Let's take the same student's sequel writing for example.

In addition, in my opinion, calligraphy is also a concrete and abstract expression. For a while, there was a debate in calligraphy circles about the characteristics of calligraphy. Some people believe that calligraphy is a figurative art, which is based on the hieroglyphics of the origin of Chinese characters and the discussion of the iconicity of calligraphy in classical books. Others believe that calligraphy is an abstract art, which is based on the symbols of words and the abstractness of line art. (excerpt) In comparison with the student's writing in Test 1 , this piece of sequel writing is much better in terms of grammar, structure, logic and diction. And there are no incorrect expressions like Chinglish in this sequel writing.

The different results of the students' two tests have a close relationship with the different requirements of the two tests and the different methods taken by the students in the course of writing.

As for the questionnaire, we got the statistical results as follows:

1 Which writing do you think is more difficult, Test 1 or Test 2?

$15 \%$ Test $1 ; 85 \%$ Test 2

2 What did you mainly refer to from the given English material in Test 2?

$75 \%$ main idea; $10 \%$ writing method; $10 \%$ words and expressions; $10 \%$ syntactic structure

3 Did you think of using the words, expressions or sentence structures written in Test 1 when you did Test 2?

$70 \%$ yes; $30 \%$ no

4 What is the main problem about your academic English writing?

$60 \%$ specialized vocabulary; $100 \%$ syntactic structure; $30 \%$ relevant knowledge

5 Do you think the sequel writing is helpful? If so, in what aspects?

$90 \%$ yes, mainly in vocabulary and syntactic structure

\section{DISCUSSION}

From the questionnaire, we know that the main problem about the students' academic English writing lies in syntactic structure (100 per cent) and specialized vocabulary (60 per cent), which means that it is difficult for students to express their ideas in exact English language forms. So, if they can improve their English language expression, they will improve their academic English writing. Statistical results showed that most students did better in Test 2, as they got the grammatical mistakes, Chinglish, and inexact diction greatly reduced. This indicates that the "continuation method" had an effect of "alignment" in the students' academic English writing. Furthermore, the "alignment" was mainly realized by language form rather than content, which also verified that "the alignment of continuation is not even for different linguistic items" ${ }^{[10]}$.

The salient advantage of "continuation method" lies in making a context of language interaction, which is conducive to reducing the adverse influence of one's native language and culture upon the language acquisition, and helping people use appropriate language forms to express their ideas. In addition, "rich contextual information promotes language understanding, language understanding promotes language alignment, language alignment promotes language output, and language output promotes language acquisition" [11]. In the course of language interaction, the linguistic and non-linguistic elements will get integrated, which regulates one's language output. That is why in the questionnaire 85 per cent students thought that Text 2 was more difficult. When they did Text 2, they had to follow the meaning and stylistic features of the given English material. They consciously borrowed or imitated the expression forms of the given English material, and accordingly adjusted their own writing style. Actually, in the course of writing, the 'structural priming' not only promoted the interaction between the students and the given English material but also the interaction between the students and their own previous writing. That is why 70 per cent students thought they used the words, expressions or sentence structures written in Test 1 when they did Test 2.

It is just by "structural priming" that the "continuation method" produces the effect of "alignment". Even though most students did better in Text 2 , there still exists a lot of room for them to improve their writing. From the questionnaire, we know that only 30 per cent students paid attention to the language expressions (writing method, words and expressions, syntactic structure) of the given English material. Most students just cared the meaning of their writing, and they did not get all their thoughts well expressed by proper language forms, so the "alignment" of "continuation" was not well realized. In addition, the "alignment" of "continuation" is also related to the students' own language aptitude. Although all students can improve their academic English writing through the "continuation method", it is often more efficient and effective for those with better language aptitude.

The "structural priming" of "continuation method" is affected by various linguistic and non-linguistic factors. For the Chinese college students, the Chinese language and culture will always influence their foreign language learning, as the use of Chinese language has formed a solidified connection with particular contexts. Besides, "the various variables, such as the cognitive, social, and psychological factors, will take effect at the same time" ${ }^{[12]}$. Therefore, although the college students have developed the abilities of reasoning, thinking and cognizance, these abilities will weaken the "alignment" of "continuation" if 
they are inconsistent with the English language and culture, as the solidified connection with the students' Chinese language and culture will get involved in the process of "alignment", making the students tend to use a "vernacularized foreign language" ${ }^{[13]}$. However, if in a good or authentic English language context, the "continuation method" can effectively reduce the negative transfer of Chinese language and culture, and thus improve the effect of English language learning.

In view of the function of "continuation method" in reducing the students' grammatical mistakes, Chinglish and inexact words and expressions, we can positively adopt it in the teaching of academic English writing for the Chinese college students. But we should pay attention to two things before using the method. First, we should take into consideration the students' language aptitude. Ensure that all given English materials are fit for their understanding ability and knowledge level. Second, we should help the students master the given English materials from two dimensions, content and form. The content helps the students know what to write, and the form helps them know how to write. The study shows that the "alignment" of "continuation method" is mostly realized by the language form, which often determines the improvement degree of students' academic English writing. After the students finish a piece of sequel writing, we should let them make an evaluation of their own compositions according to the reference writing. And then teachers should also make comments on the students' writing in general. The students' evaluation can help themselves find out why there exist differences between their writing and the reference one. Teachers' comments mainly help the students understand the strong points and shortcomings about their sequel writing, and know how to improve it. From the questionnaire, we know that 90 per cent students thought that the sequel writing was helpful to them. In addition, to achieve an ideal effect of "alignment", we should strengthen the training of students' basic language skills, as they should have necessary abilities of language understanding and production. Generally speaking, for the students who have better language skills, the effect of "alignment" is usually better.

\section{LIMITATIONS}

The limitations of the study should be addressed for future research in this area. First, as for the comparison of the students' two writing tests, it was just based on the teacher's subjective judgement and teaching experience, as the teacher knew that the "continuation method" could surely improve the students' writing, and just wanted to investigate how and at what linguistic levels the "alignment" of "continuation" was realized. So the comparison was neither systematic nor statistically quantified. In future research, this kind of comparison can be dealt with by relevant statistic software. Second, the study just proved the good effect of "continuation method" in the teaching of students' academic English writing, but it did not verify whether the "alignment" could produce a long lasting effect or not. So in future research, we should take measures to verify the actual improvement that the students have made through the "continuation method" in their academic English writing.

\section{CONCLUSIONS}

This study probed into the function of "continuation method" in improving the academic English writing of Chinese college students, and revealed that the function was realized through "structural priming", which promoted the students to actively go in for interaction with the given language material. And the outcome of "structural priming" is expressed by the effect of "alignment", which is mainly realized by language form and is related to the students' language aptitude.

Due to the gap in language proficiency between the students and the given language material, the "continuation method" stimulated the students' desire to make a constant reference to the given language. Therefore, the "continuation theory" is, in essence, a further development of the interaction theory. It highlights the importance of language use or output. In order to use the language, learners will constantly make "structural priming", getting language input and output closely connected. Thus, the efficiency of language learning will be improved.

In order to make appropriate language output, when making interaction with the given language, "learners will actively focus on language use" ${ }^{[14]}$. That is why this study shows that the effect of "alignment" is mainly manifested in the students' language expressions. In addition, the "continuation method" provides an ideal language context, which can effectively prevent the negative transfer of the students' native language, culture and other factors, so it "promotes the organic bonding of foreign language forms with proper contextual knowledge, restrains the influence of their native language, and strengthens the function of context in directly initiating language use" ${ }^{[15]}$.

In addition, for language learners, some linguistic items can produce a lasting effect of "alignment", while others just produce an instantaneous effect. Therefore, the "continuation method" should be put into use in the whole process of teaching, constantly strengthening the use of certain linguistic items. In this way, the students can make a substantial progress in their academic English writing.

\section{ACKNOWLEDGMENT}

The article is funded by the Social Science Project of Jiangsu Province, China (17YYB002) and GYPT's teaching research project (TDPY201923). 


\section{REFERENCES}

[1] Wang, Chuming, The Era Significance and Feature of Continuation Theory, Foreign Language Teaching and Research. 50(3) (2018) 422-423. DOI: https:www.cqvip.com/QK/96946X/201803

[2] [11] Wang, Chuming, Three Complex and Effective Approaches to Language Acquisition in Foreign Language Teaching, Foreign Language Teaching and Research. 43(4) (2011) 540-549. DOI: 10000429(2011)04-0540-10

[3] Wang, Chuming, Mutual Alignment and Foreign Language Teaching, Foreign Language Teaching and Research. 42(4) (2010) 297-299. DOI: https: xueshu.baidu.com/usercenter/paper

[4] [15] Wang, Chuming, Language Acquisition from Writing to Continuation, Foreign Language Teaching and Research. 49(4) (2017) 547-556. DOI: 10000429(2017)04-0547-10

[5] Ambridge, B. et al., The ubiquity of frequency effects in first language acquisition, Journal of Child Language. $\quad 42(2) \quad$ (2015) 239-273. DOI: 10.1017/S030500091400049X

[6] Bergström, K. et al., First and Second Language Acquisition in German Children Attending a Kindergarten Immersion Program: A Combined Longitudinal and Cross-Sectional Study, Language Learning. 66(2) (2016) 386-418. DOI: 10.1111/lang. 12162

[7] Kaiser, I. and G. Kasberger, Children's emerging ability to discriminate L1-varieties, First Language. 38(5) (2018) 447-480. DOI: $10.1177 / 0142723718761403$

[8] Wen, Qiufang, Developing a Theoretical System of Production-oriented Approach in Language Teaching, Foreign Language Teaching and Research. 47(4) (2015) 547-558. DOI: 1000-0429(2015)04-0547-12

[9] [14] Wang, Chuming, Promotion of Language Acquisition by Continuation, Modern Foreign Languages. 39(6) (2016) 784-793. DOI: 10036105(2016)06- 0830-12

[10] Wang, Min and Chuming Wang, The Alignment of Writing Continuation, Modern Foreign Languages. 37(4) (2014) 501-511. DOI: 1003-6105(2014)04-050112

[12] [13] Wang, Chuming, Interactions in Language
Learning, Journal of Foreign Languages. 31(6) (2008) 53-60. DOI: 1004-5139(2008)06-0053-08 